\title{
GEOMETRICAL OPTIMIZATION OF DENTAL IMPLANTS WITH REGARD TO OSSEOINTEGRATION
}

\author{
Luboš ŘehouneK*, František Denk, Aleš Jíra \\ Czech Technical University in Prague, Faculty of Civil Engineering, Thákurova 7, 16629 Prague 6, Czech \\ Republic \\ * corresponding author: lubos.rehounek@fsv.cvut.cz
}

\begin{abstract}
A newly developed reference dental implant specimen type was subjected to numerical simulations of osseointegration. The goal of these tests was to optimize the geometry of the implant so as to reduce local stress concentrations and provide a better flow of stress through the whole implant body. Conditions for osseointegration were considered when evaluating the anchoring system of the implant in regard to its placement in the human cancellous and cortical bone. Numerical simulations showed that stress concentrations occur mostly in the upper cylindrical part of the implant. By increasing the width of this cylindrical part, we were able to reduce the maximum values of von-Mises stress by $20 \%$.
\end{abstract}

KEYwORDS: Dental implant, optimization, trabecular, numerical, FEM.

\section{INTRODUCTION}

The main shortcoming of commonly manufactured dental implants is their predisposition towards early failure [1]. This unwanted factor can be attributed to uneven distribution of stresses in the implant, periimplant area and bone [4, 5]. Our main goal in developing new implants was to reduce stress concentrations occuring mainly in the upper cylindrical part of the implant, just below the abutment, while preserving the osseointegration benefits of the implant. The tested implant has been patented in the Czech Republic [6].

The numerical analysis describes the mechanical behavior of the implant in regard to mechanical loads and the corresponding biomechanical stablity in the bone tissue. The tests focus on two aspects - mechanical behavior of the implant under a prescribed mechanical load and osseointegration of the implant inside the human mandible. The first part of the analysis focuses on the osseointegration of the implant in human mandible. The aim of the second part of the tests was to determine the ultimate bearing capacity of the dental implant stem according to the ČSN EN ISO 14081 - "Stomatology - Implants - Dynamic Fatigue Testing of Intraosseous Dental Implants" standard. This paper will only focus on the first part of the tests (osseointegration) and leave the second part (mechanical tests) for further studies, as some results are already available, but additional simulations are still needed.

The analysis provides an overview of stress and deformation relations of the tested implant stem in a prescribed environment in all of its parts. This is simulated by embedding the implant stem in a block of human bone (Fig. 2 and subsequently evaluating the stress and deformation relations at surface areas of the stem and at the interfaces of the stem and bone tissue.
Numerical simulations were done in the ANSYS APDL system by incorporating previously created 3D geometries (Fig. 1) into the environment of the software. The geometry of the implant stem was created in detail at the surface areas of the implant. The internal part of the stem has been created with less detail (i.e.: without the screw-thread).

\section{Numerical Testing of OSSEOINTEGRATION}

For the purpose on numerical analysis in ANSYS APDL, we created an environment where the dental implant is fixed into the bone tissue of human mandible. The implant consists of the intraosseous stem and the implant superstructure, which represents the abutment and the crown. The connection of individual volumetric bodies is attained using rigid constraints generated by the volumetric "vglue" operations. The mesh of the model consists of quadratic tetrahedrons type SOLID 187 generated in the "smart size" mode. The material of all components of the dental implant is the titanium alloy Ti-6Al-4V.

The stem of the implant is fixed into a volumetric model of human mandible, situated according to real implantation procedures. The geometrical orientation of the ribs was arranged in the least favorable position (in regard to the results of mechanical tests). The exact geometry of the bone was generated by Boolean operations (subtracting the full body volume of the stem from the body of the bone). The connection between contact surfaces of the stem and the bone was attained by volumetric "vglue" operations. The numerical model of the mandible was then subsequently remeshed according to the new boundary conditions (Fig. 2).

The front faces of the mandibles have symmetric 

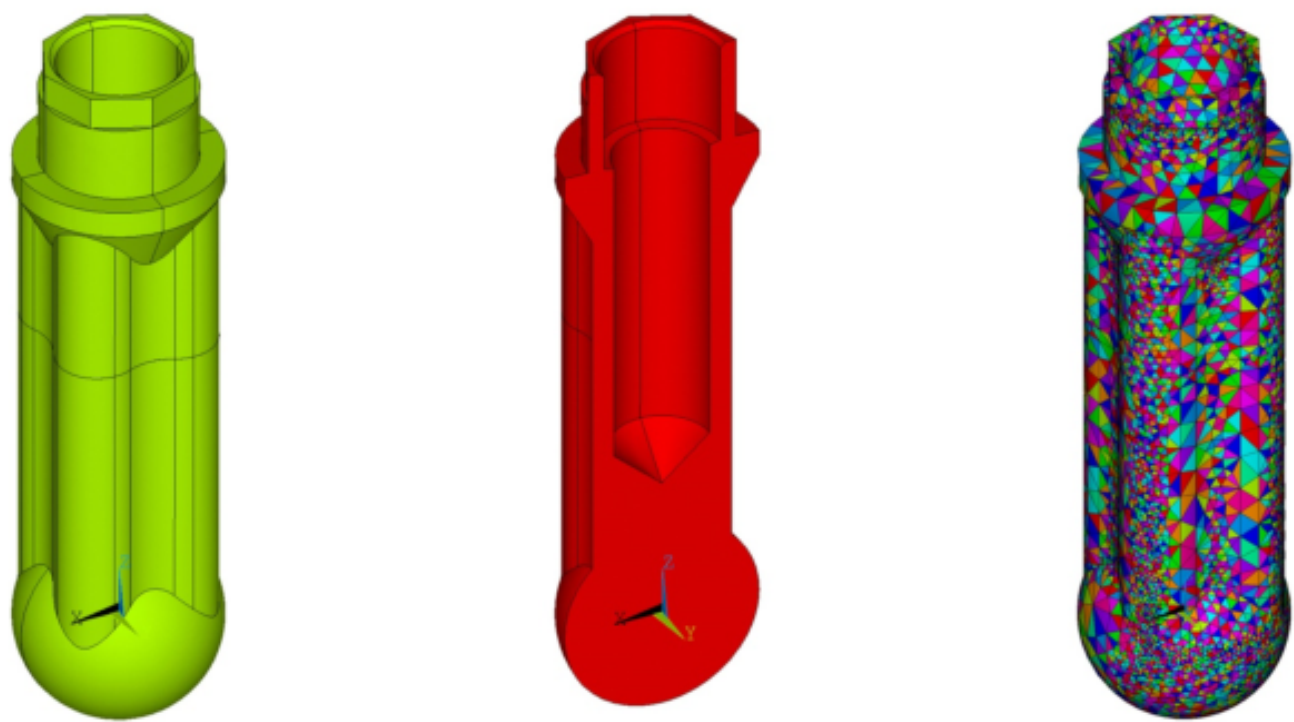

Figure 1. 3D model of the designed implant 0001 (ANSYS APDL): whole 3D model (left), longitudinal section (middle) and FEM mesh (right).
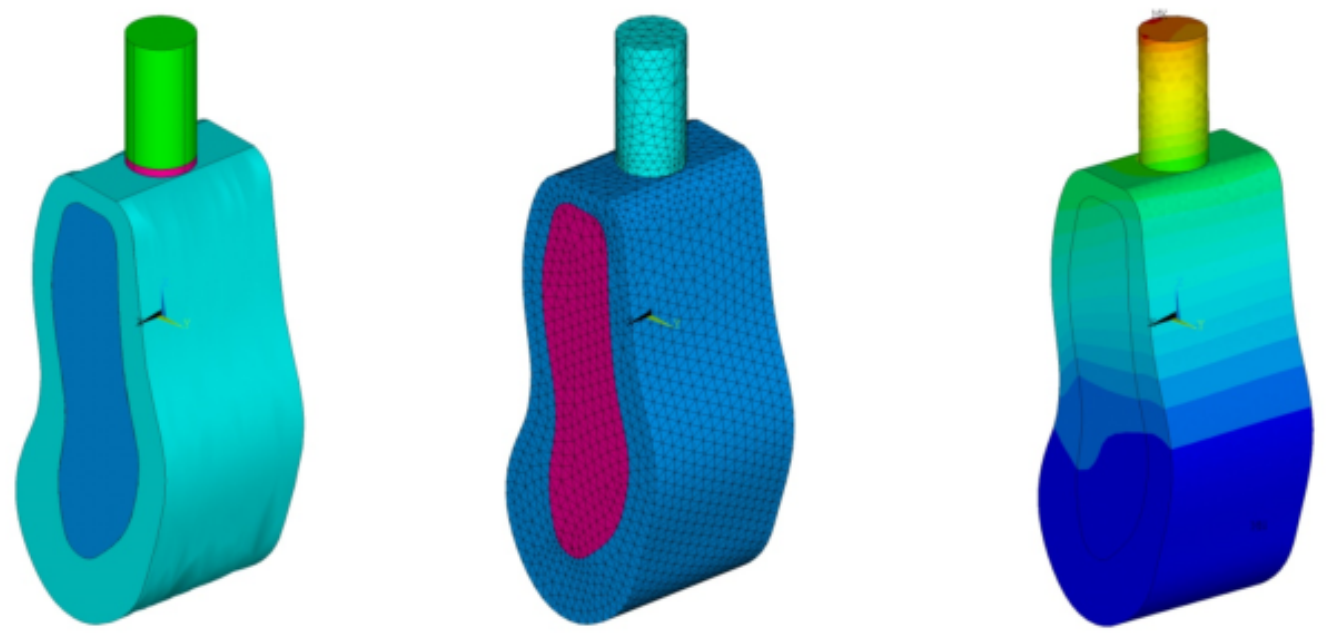

FiguRE 2. Numerical model of the mandible block which supports the intraosseous part of the implant stem volumetric model (left), FEM mesh (middle) and total deformation isolines (right).

boundary conditions and the lower strip of nodes is set to have zero displacement value. Physiological load is represented by a single slant force applied to the central point of the upper base of the superstructure. The force magnitude is $\mathrm{F}=130 \mathrm{~N}$. The direction of the force is defined by contribution of individual force components according to the total load imposed on a single tooth. The magnitude of normal force is $125 \mathrm{~N}$ and tangent force is $35 \mathrm{~N}\left(\mathrm{~F}_{\mathrm{x}}=25 \mathrm{~N}\right.$, $\left.\mathrm{F}_{\mathrm{y}}=25 \mathrm{~N}, \mathrm{~F}_{\mathrm{z}}=125 \mathrm{~N}\right)$.

\section{Optimization OF THE CylindRicAl PART}

The following optimization will assume that the small strip of the cylindrical stem forming the upper structure of the stem is always the area where values of stress concentrate. These extremities are caused
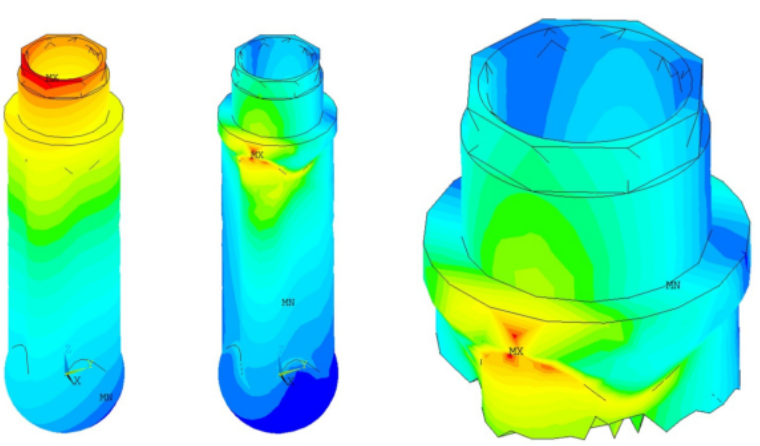

FiguRE 3. Numerical analysis of the reference implant 0001: displacement $-\mathrm{u}_{\max }=25 \mu \mathrm{m}$ (left), equivalent von Mises stress $-\sigma_{\text {eqv, } \max }=91.7 \mathrm{MPa}$ (middle and right). 

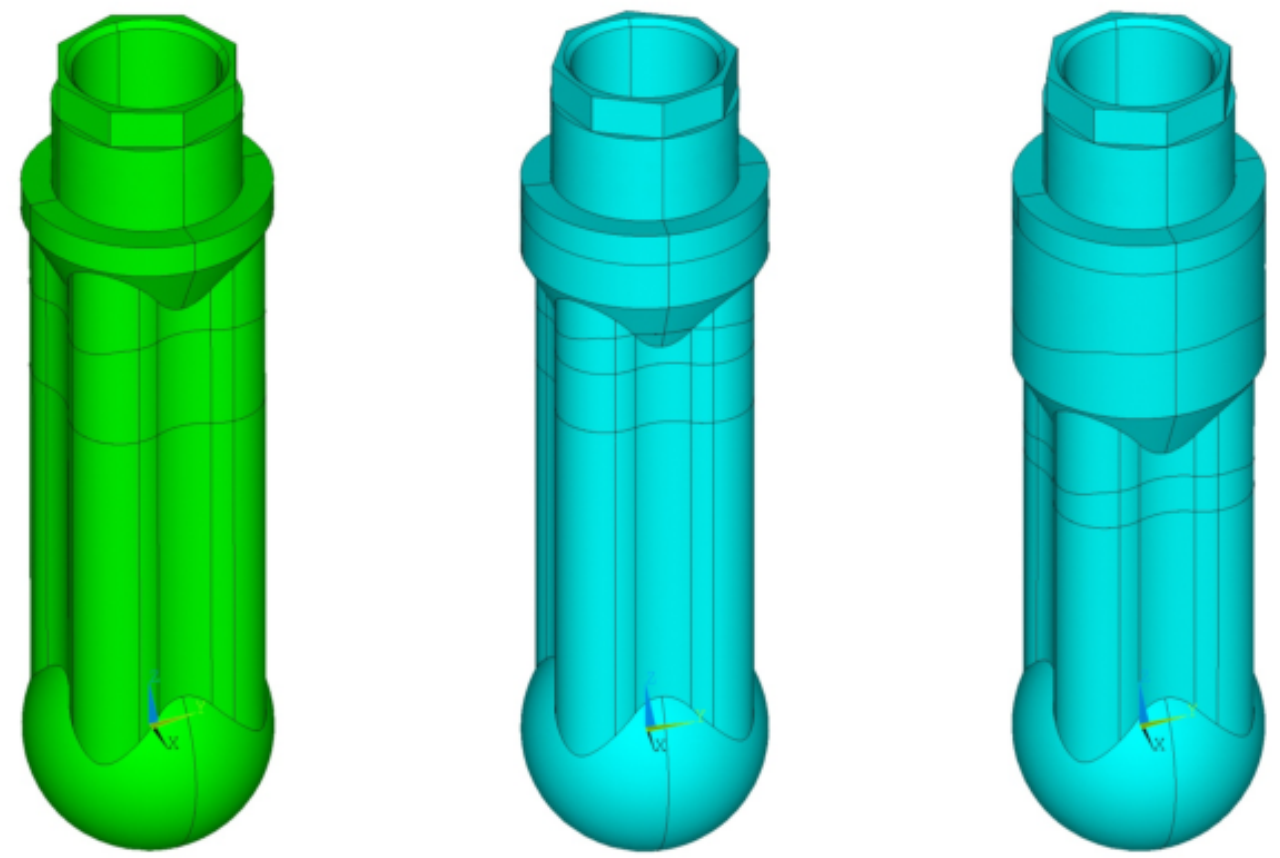

FiguRE 4. Optimization of the geometrical shape in the upper cylindrical part: reference model 0001A (cyl. width $0.50 \mathrm{~mm}$ ) (left), model 0001B (cyl. width $1.25 \mathrm{~mm}$ ) (middle) and model 0001C (cyl. width $3.00 \mathrm{~mm}$ ) (right).
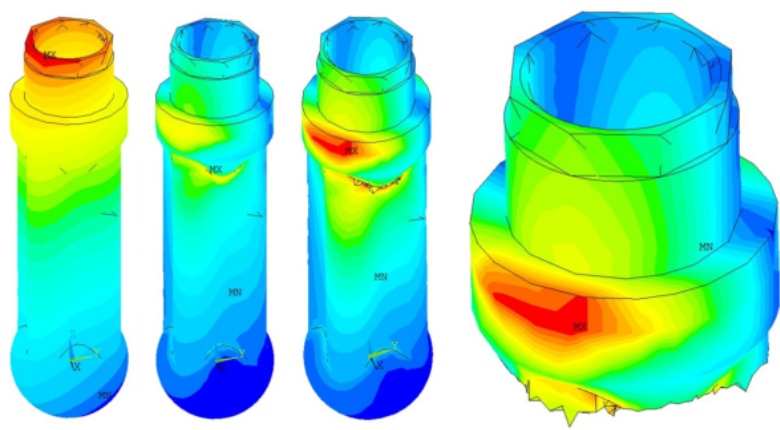

FiguRE 5. Numerical analysis of the reference implant 0001B: displacement $-\mathrm{u}_{\max }=25 \mu \mathrm{m}$ (left), equivalent von Mises stress $-\sigma_{\text {eqv }, \max , 1}=97.1 \mathrm{MPa}$, $\sigma_{\text {eqv } \max , 1}=73.2 \mathrm{MPa}$ (middle and right).

by complex geometrical shapes and also a relatively small width of the cylindrical strip. The goal of the optimization will be to effectively modify the geometrical shape of the implant in the area of fixation of the stem into cortical bone of the human mandible. Also, the overall stability of the intraosseous part must not be compromised.

Two new variants have been tested - variant 0001B and $0001 \mathrm{C}$. The modification represents increasing of the width of the cylindrical part. The width of the cylindrical part of the reference implant 0001A is $0.50 \mathrm{~mm}$. The model $0001 \mathrm{~B}$ had the width increased up to $1.25 \mathrm{~mm}$ and model $0001 \mathrm{C}$ up to $3.00 \mathrm{~mm}$.

Identical simulations were run on all of the mentioned variants and the outcomes of the numerical analyses are shown in Fig. 3. Fig. 5 and Fig. 6.

On the basis of equivalent von-Mises stress dis-
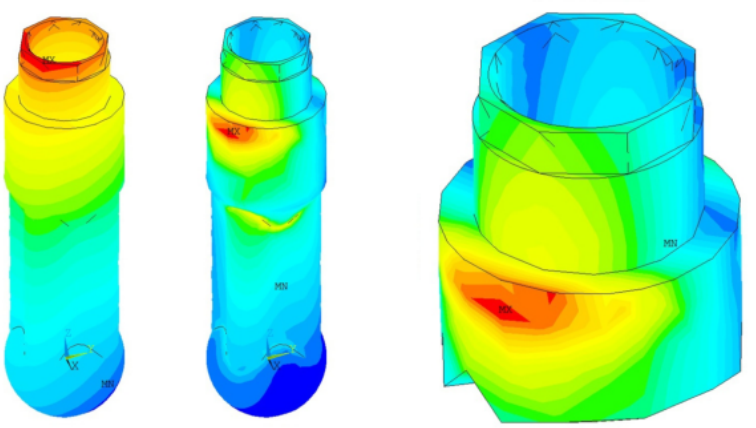

Figure 6. Numerical analysis of the reference implant 0001C: displacement $-\mathrm{u}_{\max }=25 \mu \mathrm{m}$ (left), equivalent von Mises stress $\sigma_{\text {eqv, } \max }=73.6 \mathrm{MPa}$ (middle and right).

tribution at the surface areas of the imlpant stem, we can observe that the widening of the cylindrical strip of the upper part without any other geometrical modification positively affects the stress distribution by lowering extremities.

The variant $0001 \mathrm{~B}$ (cylindrical part $1.25 \mathrm{~mm}$ ) has a mean value of $\sigma_{\text {eqv,avg }}=61.6 \mathrm{MPa}$ and the stress flow is distributed over a larger area. There is an extreme of $\sigma_{\text {eqv, } \max }=73.2 \mathrm{MPa}$. There is also another extreme of $\sigma_{\text {eqv, } \max }=97.1 \mathrm{MPa}$ spread over a very small area situated in the edge of the cylindrical part. The system of vertical ribs is situated in both cortical and cancellous bone.

The variant $0001 \mathrm{C}$ (cylindrical part $3.00 \mathrm{~mm}$ ) has a maximum value of $\sigma_{\text {eqv, } \max }=73.6 \mathrm{MPa}$, but the system of vertical ribs is situated now only in cancellous bone. 


\begin{tabular}{lccc}
\hline $\begin{array}{l}\text { Implant type } \\
\text { osseointegrated in bone } \\
\text { tissue of mandible }\end{array}$ & $\begin{array}{c}\text { Displacement of the } \\
\text { implant and bone tissue }\end{array}$ & $\begin{array}{c}\text { Equivalent } \\
\text { von-Mises stress } \\
\mathrm{u}_{\mathrm{t}}[\mu \mathrm{m}]\end{array}$ & $\begin{array}{c}\text { Optimization } \\
\text { influence }\end{array}$ \\
\hline Implant 0001A & 25 & 91.70 & 100.00 \\
Implant 0001B & 25 & 73.20 & 79.80 \\
Implant 0001C & 25 & 73.60 & 80.00 \\
\hline
\end{tabular}

TABLE 1. Overview of the results provided by geometrical modification. Reference implant 0001A and modified implants 0001B and 0001C.
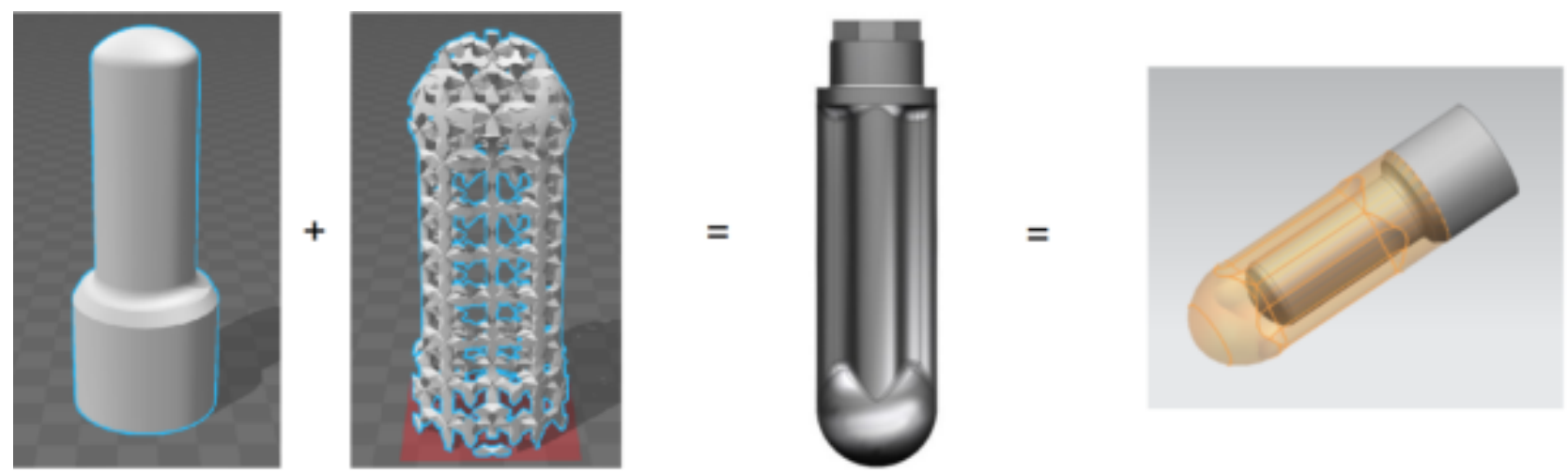

Figure 7. Two STL files showing the final geometrical solution of the implant (trabecular outer shell second from left) (left), the final implant model (middle) and homogenized layer of the outer trabecular structure for easier simulations (right).

\section{Conclusions}

\subsection{Results}

The results of the numerical simulations show that by increasing the upper cylindrical portion of the stem, we can improve the distribution of stresses at the surface areas of the implant and also reduce extreme values of stress.

The variant 0001B showed that by increasing the width of the cylindrical part by $0.75 \mathrm{~mm}$ (from $0.50 \mathrm{~mm}$ to $1.25 \mathrm{~mm}$ ), we can reduce stresses by approximately $20 \%$ and achieve a more even distribution. There is, however, an extreme value of $97.1 \mathrm{MPa}$ situated at the edge of the cylindrical part over a very small area. This extreme is caused by discontinuities in geometry (sudden change of curvature), which will be accounted for by rounding the edge of the cylinder by a radius of at least $0.1 \mathrm{~mm}$ during the production process. Biomechanical stability is achieved by situating the vertical ribs of the implant inside the cortical bone of the mandible.

The variant $0001 \mathrm{C}$ (width from $0.75 \mathrm{~mm}$ to $3.00 \mathrm{~mm}$, $2.50 \mathrm{~mm}$ increase) also showed a $20 \%$ reduction in values of stress. The cylindrical part of the implant does not permeate through the whole layer of cortical bone, so the distribution of stress is more even and there are no larger extremes in values of stress. This geometrical solution, however, might not provide sufficient biomechanical stability as the vertical ribs, which are supposed to anchor the implant in the bone, are only situated in cancellous bone.

Considering the biomechanical implications of the presented results, we evaluated the variant 0001B with a $1.25 \mathrm{~mm}$ wide cylindrical strip as the most efficient solution, which reduces extreme values of stress as well as provides sufficient stability inside thevbone tissue.

\subsection{Future Prospects}

The chosen variant 0001B has good mechanical stability and stress distribution and was chosen as the variant for further development. With the shape solution of the implant stem complete, the next step in the research will be to create a numerical model of a composite implant (Fig. 8). This implant will comprise of both homogeneous and trabecular portions. Trabecular structure has been discussed at length in one of other author's works [7].

The implant will maintain its homogeneous stem. The outer shell with the stabilizing ribs will, however, be comprised exclusively of the trabecular structure. Further simulations will be made and multiple variants of different layer thickness analysed in order to determine which shape solution is the best for osseointegration, mechanical stability and machanical bearing capacity.

The approach towards modelling the trabecular structure in future research will be focused on sustainable, repeatable analyses. We have already found this 


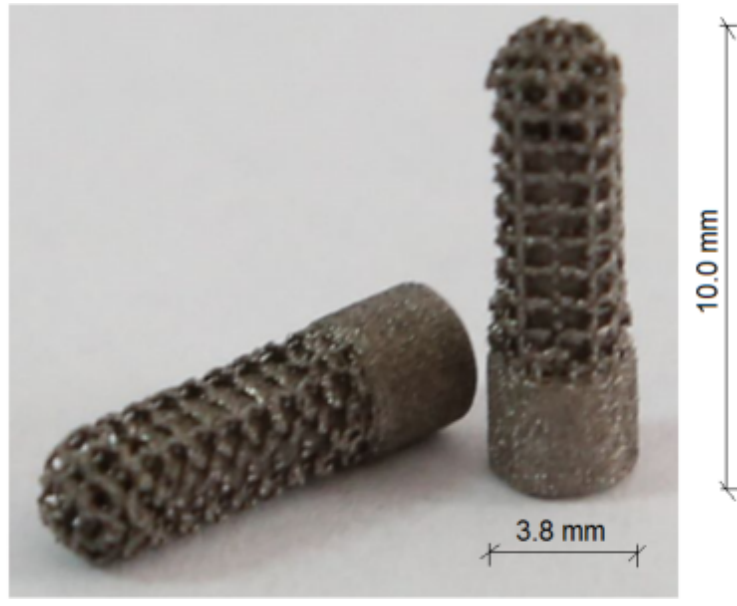

FigURE 8 . Whole implant body of the implant for further research. Implant has a homogeneous stem and an outer layer comprising of the trabecular structure. The implant has been patented in Czech Republic [6].

to only be possible by making a virtual homogenized material (Fig. 7) with specific curve-fitted properties determined by experiment [7]. This approach allows us to apply previously determined mechanical properties of the trabecular structure onto a homogeneous material that is easy to analyze in numerical environment. By running a few simple analyses, we found this to be the optimal approach as it is not very demanding in computation times and it maintains sufficient precision. By manipulating the shape of the outer trabecular structure, we will then be able to determine which variant is optimal in regard to reduction of stress shielding and mechanical bearing capacity.

\section{ACKNOWLEDGEMENTS}

The financial support by the Faculty of Civil Engineering, Czech Technical University in Prague (SGS project No. SGS17/168/OHK1/3T/11) is gratefully acknowledged.

\section{REFERENCES}

[1] L. Le Guéhennec, A. Soueidan, P. Layrolle, Y. Amouriq. Surface treatments of titanium dental implants for rapid osseointegration. Dental Materials 23(7):844-854, 2007. DOI:10.1016/j.dental.2006.06.025.

[2] R. Osman, M. Swain. A Critical Review of Dental Implant Materials with an Emphasis on Titanium versus Zirconia. Materials 8(3):932-958, 2015. DOI:10.3390/ma8030932

[3] T. Jemt, M. Olsson, F. Renouard, et al. Early Implant Failures Related to Individual Surgeons: An Analysis Covering 11,074 Operations Performed during 28 Years. Clinical Implant Dentistry and Related Research 18(5):861-872, 2016. DOI:10.1111/cid.12379

[4] M. I. Z. Ridzwan, S. Shuib, A. Y. Hassan, et al. Problem of stress shielding and improvement to the hip implant designs: A review, 2007. DOI:10.3923/jms.2007.460.467

[5] M. Long, H. J. Rack. Titanium alloys in total joint replacement-a materials science perspective. Biomaterials 19(18):1621-1639, 1998. DOI:10.1016/S0142-9612(97)00146-4

[6] F. Denk, F. Denk, A. Jíra. A dental implant shaft, Czech Republic, 306457, 2017.

[7] L. Řehounek, A. Jíra. Experimental and numerical analyses of a 3D-printed titanium trabecular dental implant. Acta Polytechnica 57(3):218-228, 2017. DOI:10.14311/AP.2017.57.0218 